\title{
Darmstädter Symposium Informationswissenschaft
}

\section{Tilman Deuschel, Timm Heuss und Bernhard Humm, Darmstadt \\ Die Medienplattform: Ein System für gerichtete und ungerichtete semantische Suchen}

DOI 10.1515/iwp-2015-0046

Die Medienplattform bietet neuartige Möglichkeiten, um Medienbestände zu entdecken. In zwei verschiedenen Anwendungsszenarien unterstützt sie unterschiedliche Suchparadigmen: Für browsingbasierte Anwendungen wird das Digitale Schlendern vorgestellt, eine Methode in der semantische Zusammenhänge von Medien als Explorationsanreiz genutzt werden. Für Anwendungen bei denen der Nutzer weiß, was er sucht, aber nicht wie das gesuchte Medium heißt, wird eine suchkontextsensitive semantische Facettierung vorgestellt.

Deskriptoren: Internetdienst, Multimedial, Suchhilfe, Benutzerfreundlich, Datenstruktur, Visualisierung, Museum, Katalog, Informationsanalyse, Digitales Schlendern

\section{The Mediaplatform: A system for directed and} undirected semantic searches

The Mediaplatform offers new possibilities to explore media collections. With two different usage scenarios, the platform supports two distinct search paradigms: for browser-based applications, we introduce Digital Strolling - a new method to stimulate exploration using the semantic connection within the collection. For applications where the user has a clearer perception of his information needs, we introduce a context-sensitive, facetted semantic search.

Descriptors: Internet service, Multimedia, Search aid, User friendly, Data structure, Visualization, Museum, Catalog, Information analysis, Digital strolling

La plate-forme de médias: un système de recherches sémantiques directionnelles et non directionnelles

La plate-forme de médias offre des nouvelles façons de découvrir des fonds de médias. Elle soutient deux paradigmes de recherche différents selon le scénario d'application: pour les utilisations basées sur le browsing, elle propose la promenade numérique, une méthode qui utilise les contextes sémantiques des médias comme incitation à l'exploration. Pour les utilisations où l'utilisateur sait ce qu'il cherche mais ne connait pas le nom du média recherché, la plate-forme propose une recherche sémantique contextuelle qui fait appel à des facettes.

Descripteurs: Service de l'internet, Recherche de l'information, Multimédia, Visualisation, Structure des données, Musée, Catalogue, Analyse de l'information, Promenade numérique

\section{Einleitung}

Wer einmal vor Sandro Botticellis „Weibliches Idealbildnis“ gestanden hat, weiß, wie sehr dieses Meisterwerk, aber auch dessen Rahmung, die Ausleuchtung und der Farbton des umgebenden Raumes, und viele andere, zum Teil subtile Sinneseindrücke eine einzigartige Atmosphäre schaffen. So wird das Bildnis zu einem Erlebnis - und genau das kann man digital nicht nachbilden. Doch man war möglicherweise aus Neugierde versucht, die Stimmung oder dargestellte Mode des Portraits mit der anderer Epochen zu vergleichen - interessante Bilder zum Vergleich einfach einmal nebeneinander zu betrachten. Oder sich das gesamte Spektrum zu betrachten, wie Personen unabhängig von Stil oder Zeit im Bestand des $\mathrm{Mu}$ seums dargestellt wurden. Hier stößt man auf die Grenzen eines jeden physikalischen Museums, und auf nur eine der vielen Möglichkeiten im digitalen Raum.

Mit der Medienplattform präsentieren wir ein System zur neuartigen Vermittlung von Medien, um Online-Kataloge von Galerien, Bibliotheken, Archiven und Museen im Web von heute zu repräsentieren - ohne physische Erfahrungen nachzubauen. In diesem vom Land Hessen geförderten Projekt kooperierten der Fachbereich Informatik und der Fachbereich Media der Hochschule Darmstadt gemeinsam mit dem Museum Städel, dem Hessischen Bibliotheks- und Informationssystem (HeBIS), der Universitätsund Landesbibliothek Darmstadt und den Industriepart- 
nern House of IT, mediatransfer AG und Software AG. Umgesetzt wurden zwei unterschiedliche Anwendungen mit hohen Anforderungen an Nutzerfreundlichkeit, Geschwindigkeit, Korrektheit und Stabilität. Beide arbeiten in der gemeinsamen Architektur der Medienplattform, die riesige Datenbestände aus heterogenen Quellen miteinander kombiniert und nutzungoptimiert ausliefert.

Die zwei unterschiedlichsten Szenarien, aus denen sich die Anforderungen für eine Informationssuche ableiten lassen, sind die gerichtete und die ungerichtete $\mathrm{Su}$ che, die jeweils einen Anwendungsfall für Museum, bzw. Bibliothek darstellen. In einem Museumsbestand steht das lustvolle Explorieren im Vordergrund. Es müssen den Nutzerinnen und Nutzern kontinuierlich Möglichkeiten aufgezeigt werden, den Bestand weiter zu entdecken. Dagegen haben Nutzerinnen und Nutzer eines Bibliothekbestands eine mehr oder weniger gut formulierte Zielvorstellung: in möglichst wenigen Schritten soll das gefunden werden, was gesucht wurde. Insbesondere bei unpräzisen Anfragen muss ebenfalls eine Unterstützung erfolgen - allerdings mit dem Ziel einer Verdichtung.

Im Folgenden werden die beiden unterschiedlichen Nutzungsszenarien genauer beschrieben und die individuellen Anforderungen ausgeführt. Danach beschreiben wir die Architektur, die dies performant und in Hinblick auf modernen Paradigmen der Webentwicklung ermöglicht.

\section{Semantische Suche mit der Medienplattform}

Das Suchverhalten von Nutzern ist komplex und von vielen Randbedingungen abhängig [9], insbesondere von Nutzerbedürfnissen. Daher wird durch User Centered Design Funktion und Form von den Anforderungen abgeleitet [13].

Die gerichtete Suche steht für ein konkretes Informationsbedürfnis, das nur durch sehr spezifische Ergebnisse befriedigt werden kann. Der Nutzer weiß wann die Suche erfolgreich abgeschlossen ist [9], er kennt jedoch unter Umständen die Metadaten der gesuchten Information nicht, wie z. B. Autor und Titel. Für Informationsportale, die die gerichtete Suche unterstützen, wie der Anwendungsfall Bibliothek, werden Filtersysteme genutzt, die Ergebnisse der aktuellen Anfrage einschränken.

Bei der ungerichtete Suche, also Browsen, besteht auch ein Informationsbedürfnis, jedoch kann dieses durch sehr verschiedenartige Ergebnisse befriedigt werden, weil der Nutzer keine spezifischen Ziele hat [1]. Bei Informationsportalen, die die ungerichtete Suche unterstützen, wie der Anwendungsfall Museum, werden dem
Nutzer Navigationsvorschläge für neue Inhalte angeboten [8]. In der Realität wechseln Nutzer häufig ihre Suchstrategien, teilweise auch unbewusst [9].

\section{Anwendungsfall Museum}

Die Nutzungsoberfläche für den Anwendungsfall Museum ist darauf ausgelegt, eine Navigation über Inhalte, statt über eine abstrakte Navigationsstruktur anzubieten. Als Einstieg wird dem Nutzer eine Auswahl von Bildern von Kunstwerken präsentiert. Wählt er ein Bild aus, werden ihm weitere Bilder angeboten, die in einem Zusammenhang zu dem aktuell betrachteten Werk stehen. So kann der Nutzer über verschiedene Dimensionen hinweg seinen Weg durch den Bestand der digitalen Kunstwerke beschreiten, ein Prozess, den wir Digitales Schlendern nennen, wie schematisch in Abbildung 1 dargestellt. Die Dimensionen, die vom aktuell betrachteten Werk zu weiteren Werken verlinken, beziehen sich sowohl auf inhaltliche Gemeinsamkeiten als auch auf emotionale. Eine ähnliche Funktion bieten sogenannte Image RetrievalSysteme. Diese sind jedoch nur auf inhaltliche Gemeinsamkeiten spezialisiert [2]. Emotionale Zusammenhänge von z. B. ähnlicher Atmosphäre, Bildwirkung und Assoziationen sind im Bereich Cultural Heritage nach bestem Wissen der Autoren noch nicht in vergleichbarer Qualität der intellektuellen Verschlagwortung eingeführt worden.

Die Schlendervorschläge werden nun anhand der Anzahl der gemeinsamen, normierten Schlagworte generiert. Abbildung 2 zeigt, dass das aktuell betrachtete Werk (Mitte) mehr gemeinsame Tags in der Dimension Emotion zu dem Werk auf der rechten Seite, als auf der linken Seite aufweist und wird daher als relevanter Schlendervorschlag für die gegebene Dimension angeboten.

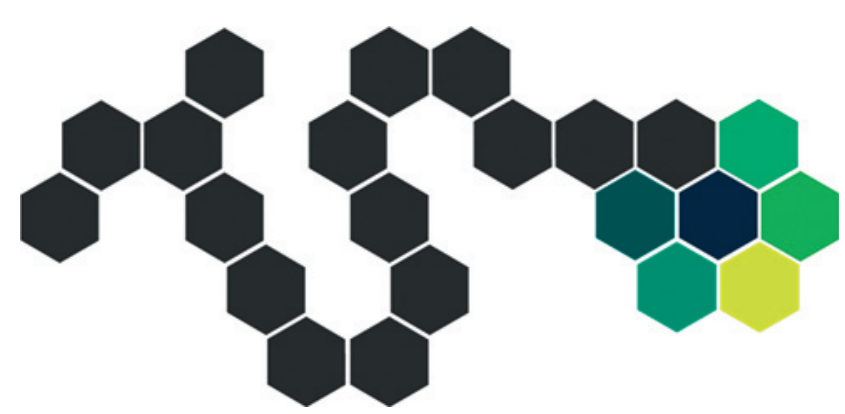

Abbildung 1: Schematische Darstellung des Digitalen Schlenderns. Die dunkelgrauen Kacheln stellen den Pfad dar, den ein Nutzer durch die verschiedenen semantisch zusammenhängenden Werke gegangen ist. Die um das momentan betrachtete Werk (dunkelblaue Kachel) gruppierten Kacheln sind Schlendervorschläge, die einen semantischen Bezug aufweisen. 

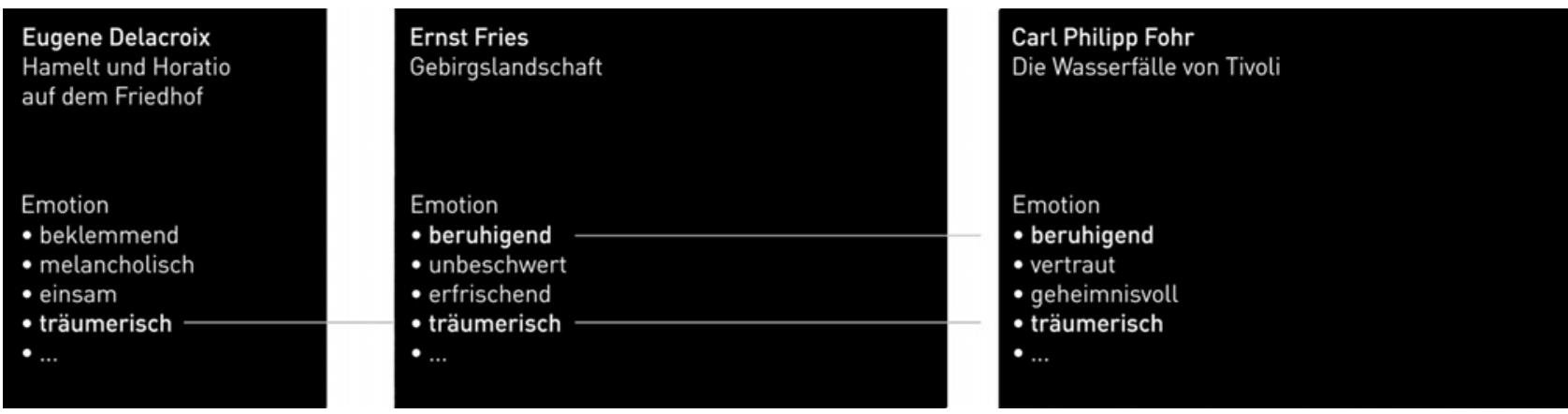

Abbildung 2: Das aktuell angezeigte Werk (Mitte) weist eine größere Ähnlichkeit in der Dimension „Emotion“ zu dem Werk auf der rechten Seite auf, weil dieses mehr gemeinsame Tags in der gegebenen Dimension zu dem aktuell betrachteten Werk aufweist als das auf der linken Seite. Daher wird das Werk zur Rechten als Schlendervorschlag für das Bild in der Mitte in der Dimension „Emotion“ angeboten.

\section{Anwendungsfall Bibliothek}

Laut einer Umfrage zur Verbesserung des HeBIS Katalogs empfinden 84 Prozent der über 21.000 Befragten eine Filterung der Ergebnisse als wichtige Funktion [10]. Eine facettierte Suche bietet eine solche Filterung, die dem Nutzer durch ihre Strukturierung der Ergebnisse nicht nur Zeit spart [7], sondern auch die Exploration unbekannter Domänen anregt [12]. Facetten, wie sie häufig in Library Discovery Services genutzt werden, weisen Schwächen auf, z. B.: ist die Facette selten dynamisch an die aktuelle Suchergebnismenge angepasst oder sie ist zu flach. Dadurch wird die Ergebnismenge nicht auf eine überschaubare Anzahl reduziert und damit ihre Funktion nicht erfüllen. Im Anwendungsfall Bibliothek wird dieses Problem

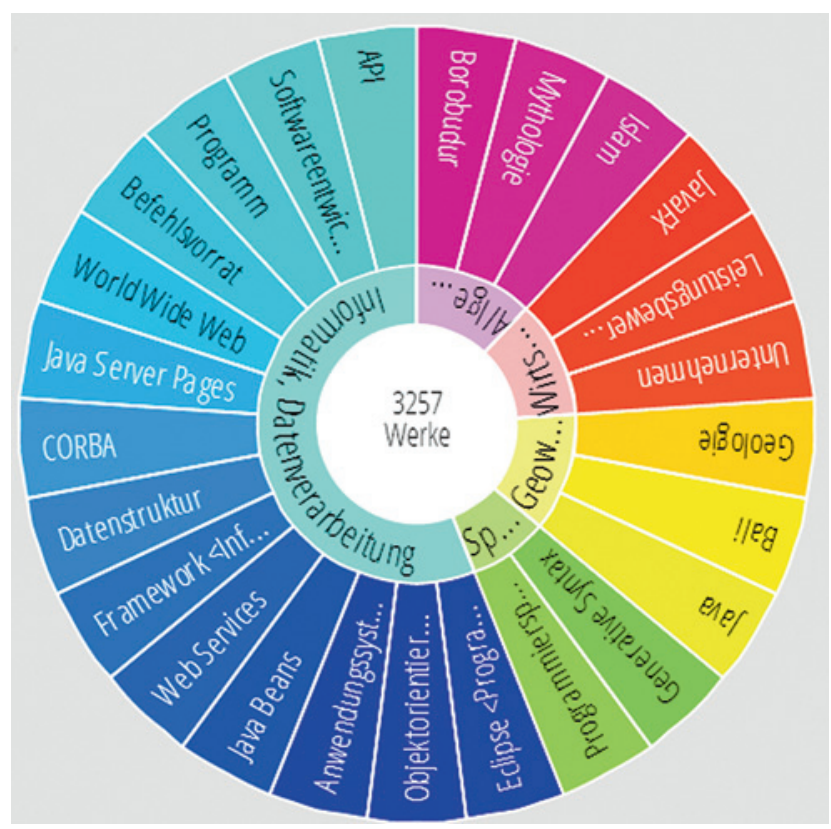

Abbildung 3: Für Touchinput optimierte facettierte Suche des Anwendungsfalls Bibliothek. durch eine kontextsensitive Facette, die die Suchergebnismenge in der Dimension der Schlagworte der Werke unterteilt, gelöst. Sucht der Nutzer, z. B. nach „Java“ bietet die Facette Kategorien wie „Informatik“, „Sprache“, „Geowissenschaften“, etc. an. Jede Kategorie bietet Ausprägungen, wie z. B. „Informatik: API“ oder „Geowissenschaften: Bali“, in einem drehbaren Kreis, Themenrad genannt, an, siehe Abbildung 3. Im inneren Kreis sind die Kategorien, im äußeren die Ausprägungen angeordnet. Die Kontextsensitivität wird dadurch erreicht, dass die Schlagworte der Werke, die in der Ergebnismenge enthalten sind mit Hilfe der Gemeinsamen Normdatei (GND) der Deutschen Nationalbibliothek hierarchisch strukturiert werden. So werden semantisch zusammengehörende Themenfelder unter einem Oberbegriff zusammengefasst, der wiederum nicht zu generisch gewählt sein darf, um den Nutzer einen Nutzen erahnen zu lassen. Wird eine Verfeinerung gewählt, ändern sich die Ergebnismenge und damit der Kontext, in dem dieses Optimierungsproblem erneut gelöst werden muss, mit dem Ergebnis, dass die Verfeinerungsvorschläge spezifischer werden. Da die Filterung konjunktiv verknüpft durchgeführt wird, kommt ein Nutzer, der nur den Themenbereich kennt, aber nicht Autor oder Titel, so mit wenigen Klicks zu einer überschaubaren Anzahl von Werken.

\section{Architektur der Plattform}

Der Nutzer im Web von heute ist an Geschwindigkeit gewöhnt: Alles, was nicht quasi sofort dargestellt wird, wird schnell durch sinkende Nutzerzahlen bestraft [11]. Daher müssen Performance und zielgruppengerechte Datenauslieferung bereits zu Beginn berücksichtigt werden und ein zentrales Entwicklungsleitbild der Architektur sein. Dies zeigt sich auch bei anhaltenden Paradigmen wie der Polyglot Persistence, wo für unterschiedliche An- 


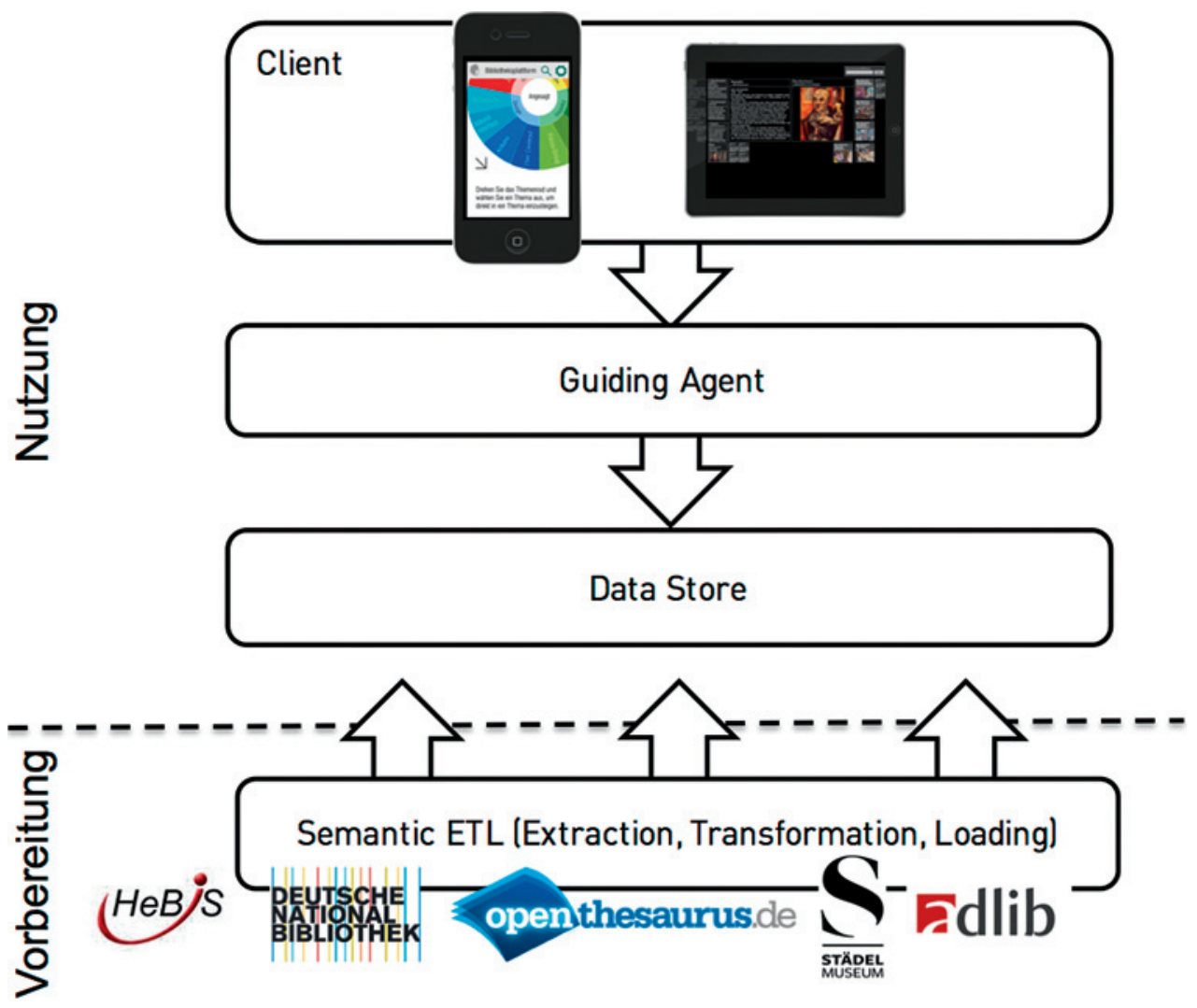

Abbildung 4: Die Schichtenarchitektur der Medienplattform.

wendungsfälle einer Anwendung die jeweils am besten geeignete Datenbank gewählt wird [5], S. 381.

Entsprechend ist die Architektur der Medienplattform stark anforderungsgetrieben entworfen. Die unterschiedlichen Schichten sind in Abbildung 4 dargestellt. Es unterscheiden sich grundsätzlich Datenauf- und -vorbereitung (unterhalb der gestrichelten Linie), welche offline geschieht, und deren Nutzung im Live-Betrieb (oberhalb der gestrichelten Linie).

\section{Semantic ETL}

In Anlehnung an die Datenintegration in Datenbanken heißt der Prozess der Datenvorbereitung, -integration, -anreicherung und -optimierung der Medienplattform Semantic Extraction, Transform, Load (ETL). Dabei werden aus unterschiedlichen Quellen Medienbestände, wie Bücher aus dem HeBIS-Katalog und Exponate des Museums Städel, um taxonomische Informationen und Expertenschlagworte ergänzt, in ein gemeinsames Datenmodell überführt und im Hinblick auf die oben genannten fachlichen Nutzungsszenarien hin optimiert gespeichert.

In dem Prozess wird neben dem Bestand des Museums Städel auch (Linked) Open Data wie etwa der He-
BIS-Katalog, die GND und OpenThesaurus in vielen unterschiedlichen Dateiformaten (XML, RDF, MARC, Plain Text, Microsoft Excel) verarbeitet. Der gewählte Prozess führt zudem Schema-basierende Entitäten ein - im Gegensatz zu der typischen Vorstellung in Semantic-WebSystemen, die generell eher als Schema-agnostisch zu bezeichnen sind. So existiert in allen nachfolgenden Schichten der Architektur eine genaue Definition darüber, was die Entitäten der Medienplattform sind und wie sie aufgebaut sind.

\section{Data Store}

Sowohl für die Indexierung als auch für die Speicherung nutzt die Medienplattform die Suchmaschine Apache Lucene, denn beide Anwendungsszenarien lassen sich sehr effizient als Information Retrieval-Probleme abbilden [4]. Die wenigen Relationen in den Daten werden flach und denormalisiert gespeichert. Darüber hinaus kommt keine andere Speichertechnologie wie etwa eine relationale Datenbank zum Einsatz. Im Ergebnis gipfeln bisherige Optimierungsschritte in einer Speicherschicht, die übliche fachliche Anfragen über zehn Millionen Bücher und Exponate im Bereich weniger Millisekunden verarbeiten kann. 


\section{Guiding Agent}

Durch die vorausgehenden Verarbeitungsschritte können in der Guiding Agent-Schicht fachliche Probleme in der spezifischen Sprache der Anwendung gelöst werden, was zu schlanken, effizienten, problemzentriertem Programmieren führt. In dieser Schicht ist das digitale Schlendern implementiert sowie die Nutzung des Themenrads.

\section{Client}

In modernen Web-Anwendungen arbeitet man mit HTML5 und JavaScript-Frameworks, die MVC-ähnliche Paradigmen für Web-Anwendungen umsetzen. Damit wird eine lose Datenbindung der View-Komponente an Datenmodelle ermöglicht. Hier können die durch den Semantic ETL-Prozess angezogenen Daten dank der fachlichen Schnittstelle nahtlos und entwicklerfreundlich wieder verwendet werden. Die Clients für beide Anwendungsfälle nutzen eine gemeinsame fachliche Schnittstelle.

\section{Resümee}

Wir haben die Medienplattform vorgestellt, die neue Möglichkeiten bietet, Medienbestände digital $\mathrm{zu}$ entdecken. Dabei gehen wir auf die speziellen Anforderungen unserer Partner und damit unterschiedlichen Suchszenarien ein. Geschwindigkeit und Usability sind grundlegende Entwicklungsziele unserer Architektur, die große Mengen an Daten aus heterogenen Quellen und Formaten nutzbar macht und auf bisher einzigartige Weise miteinander kombiniert.

Der Museumsteil der Anwendung wurde im Kontext des 200-jährigen Jubiläums des Museums Städel mit großem Medienecho gestartet und kann seither live unter http://digitalesammlung.staedelmuseum.de betrachtet werden. Erste Testergebnisse der Bibliotheksanwendung zeigen, dass der anvisierte Explorationseffekt in der Praxis funktioniert und Nutzer auf ihrer Suche unterstützt werden [3]. Beide Projekte erhielten den Hessischen Forschungspreis 2014 [14].

Im Fokus aktueller Forschung stehen Weiterentwicklungen, um das Nutzererlebnis in der Medienplattform weiter zu verbessern. Dazu gehört der Brückenschlag zwischen Museum und Besucher durch die Möglichkeit, eigene Tags zu den Exponaten zu vergeben, sowie die Frage, wie die individuelle Erfahrung durch eine automatische Personalisierung intensiviert werden kann.

\section{Literatur}

[1] O. d. Bruijnand R. Spence. A new framework for theory-based interaction design applied to serendipitous information retrieval. ACM Transactions on Computer-Human Interaction, 15(1):1-38, 2008.

[2] G. Ciocca, P. Olivo, and R. Schettini. Browsing museum image collections on a multi-touch table. Information Systems, 37(2):169-182, 2012.

[3] T. Deuschel, C. Greppmeier, B. Humm, and W. Stille. Semantically faceted navigation with topic pies. In H. Sack, A. Filipowska, J. Lehmann, and S. Hellmann, editors, Proceedings of the 10th International Conference on Semantic Systems, pages 132-139, New York, NY, USA, 2014. ACM.

[4] T. Deuschel, T. Heuss, and B. Humm. The digital online museum. Proceedings of the 4th International Workshop on Semantic Digital Archives (SDA). Collocated with the ACM/ IEEE Joint Conference on Digital Libraries, London, 2014.

[5] S. Edlich, A. Friedland, J. Hampe, and B. Brauer. NoSQL: Einstieg in die Welt nichtrelationaler Web-2.0-Datenbanken. Hanser: München, 2., aktualisierte und erw. Aufl., 2011.

[6] T. Heuss, B. Humm, T. Deuschel, T. Fröhlich, T. Herth, and O. Mitesser. Semantically guided, situation-aware literature research, 2013.

[7] B. Kules, R. Capra, M. Banta, and T. Sierra. What do exploratory searchers look at in a faceted search interface? In F. Heath, M. L. Rice-Lively, and R. Furuta, editors, Proceedings of the 9th ACM/IEEE-CS joint conference on Digital libraries, pages 313-322, New York, NY, USA, 2009. ACM.

[8] H. Liebermann. Letizia: an agent that assists web browsing. In C. S. Mellish, editor, IJCAI'95 Proceedings of the 14th international joint conference on Artificial intelligence, volume 1, pages 924- 929, San Francisco, CA, USA, 1995. Morgan Kaufmann Publishers Inc.

[9] P. Morville and J. Callender. Search patterns. O’Reilly Media, Sebastopol, CA, USA, 1 edition, 2010.

[10] H. Nienerza, B. Sunckel, and B. Meier. Unser Katalog soll besser werden! Kataloge und Portale im Web-2.0-Zeitalter. Ergebnisse einer Online-Umfrage im Hebis-Verbund. ABITechnik, 31(3), 2011.

[11] E. Schurman and J. Brutlag. The User and Business Impact of Server Delays, Additional Bytes, andHTTP Chunking in Web Search Presentation, 2009.

[12] X. Zhang, Y. Qu, C. L. Giles, and P. Song. Citesense: supporting sense making of research literature. In M. Czerwinski, A. Lund, and D. Tan, editors, Proceeding of the twenty-sixth annual CHI conference, pages 677-680, 2008.

[13] Garrett, Jesse James (2011): The elements of user experience. User-centered design for the Web and beyond. 2. Edition. Berkeley, CA: New Riders (Voices that matter).

[14] Khakzar, Karim (2014): Verleihung des Hessischen Forschungspreises 2014. Online verfügbar unter: http://www.fh-fulda.de/ index.php?id=12870 [29.05.2015]. 


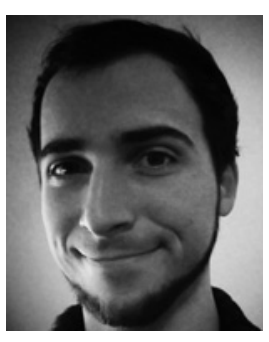

Tilman Deuschel, M. A.

Hochschule Darmstadt

Fachbereich Media

Max-Planck-Straße 2

64807 Dieburg

tilman.deuschel@h-da.de

Tilman Deuschel ist Doktorand am Cork Institute of Technology, Irland. Er erforscht User Experience von Adaptive User Interfaces, eine Form von Graphischen Nutzeroberflächen, die sich automatisch personalisieren. Davor beschäftigte er sich mit Requirement Engineering, Design, Prototypisierung und Usability von interaktiven Medien.

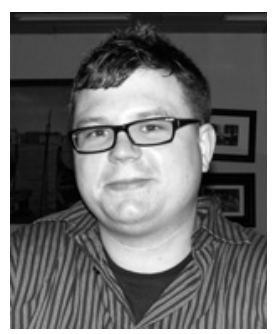

Timm Heuss, M.Sc.

Hochschule Darmstadt

Fachbereich Informatik

Schöfferstraße 8b

64295 Darmstadt

timm.heuss@h-da.de

Timm Heuss ist Ph.D. Student an der Plymouth University, England. Sein Forschungsinteresse liegt in der Entwicklung realitätstauglicher Architekturen für die Nutzung von Open und Linked Data. Zuvor arbeitete er in den Bereichen Process Management, Business Intelligence, Social Web, Semantic Web und Natural Language Processing.

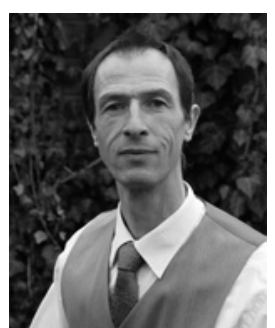

Prof. Dr. Bernhard Humm

Hochschule Darmstadt

Fachbereich Informatik

Schöfferstraße 8b

64295 Darmstadt

bernhard.humm@h-da.de

Prof. Dr. Bernhard Humm ist Professor für Software Engineering und Projektmanagement am Fachbereich Informatik der Hochschule Darmstadt und Direktor des Instituts für Angewandte Informatik Darmstadt (aIDa). Seine Forschungsschwerpunkte liegen in den Bereichen Semantic Web, Software Architektur und Programmiersprachen. 